\title{
Modelos neuronales pulsantes adaptados para el mejoramiento de luminosidad de imágenes cerebrales de gran resolución
}

\author{
Manuel Mejia-Lavalle ${ }^{1}$, Kevin Aguilar ${ }^{1}$, Humberto Sossa ${ }^{2}$, Dante Mujica ${ }^{1}$, \\ Andrea Magadan ${ }^{1}$ \\ ${ }^{1}$ Tecnológico Nacional de México / CENIDET, Cuernavaca, Morelos, México \\ ${ }^{2}$ Instituto Politécnico Nacional / CIC, Ciudad de México, México \\ \{mlavalle, kevin.aguilar17ca, dantemv, magadan\}@cenidet.edu.mx, \\ hsossa@cic.ipn.mx
}

\begin{abstract}
Resumen. En este artículo se proponen, implementan y experimenta con dos Redes Neuronales Pulsantes basadas en el Modelo de Intersección Cortical para mejorar la luminosidad en imágenes médicas del cerebro humano. Las imágenes digitales son ampliamente utilizadas en el área de medicina, pero éstas pueden ser degradadas por diversos factores. La degradación en su luminosidad genera un problema para su correcto análisis, ya que tienen un rango dinámico corto y bajo contraste. La necesidad de obtener imágenes de buena calidad y la tendencia del aumento en la resolución de éstas, exigen nuevas técnicas para mejorar la calidad en menor tiempo. Por eso es necesario buscar paradigmas que puedan aprovechar el cómputo en paralelo como los son las Redes Neuronales Artificiales Pulsantes o de Tercera Generación. La experimentación realizada muestra que los métodos propuestos son altamente competitivos comparados contra otras técnicas mencionadas en la literatura especializada.
\end{abstract}

Palabras clave: redes neuronales artificiales, redes neuronales pulsantes, modelo de intersección cortical, mejoramiento de imágenes cerebrales, imágenes de gran resolución.

\section{Pulsed Neural Models Adapted for the Enhancement of High Resolution Brain Images Luminosity}

\begin{abstract}
In this paper it is propose, implement and experiment with two Pulsed Neural Networks based on the Intersection Cortical Model for the enhancement of human brain medical images luminosity. Digital images are widely used in the medicine area, but these can be degraded by several factors. The degradation in its luminosity generates a problem for its correct analy sis, since they have a short dynamic range and low contrast. The actual necessity to obtain good quality images joined to the resolution increase tendency, demand new techniques to improve the quality, but in less time. That is why it is necessary to search for paradigms that can take advantage of parallel computing such as Pulse-Coupled Artificial Neural Networks. Experimentation shows that the proposed methods are highly competitive compared versus other techniques mentioned in the specialized literature.
\end{abstract}


Keywords: artificial neural networks, pulsed neural networks, intersection cortical model, brain images enhancement, high resolution images.

\section{Introducción}

\subsection{Motivación - problemática}

Las imágenes digitales son utilizadas en diversas áreas, una de ellas es el área de la medicina, que utiliza una gran variedad de estudios por imágenes como lo son los estudios de resonancia magnética. Sin embargo, la calidad de las imágenes puede verse afectada por muchos factores. Estas afectaciones dificultan el análisis o cualquier otro proceso que se requiera realizar con la imagen. La calidad de una imagen es descrita por las siguientes características: nitidez, contraste, color, claridad y artefactos [1]. La mala luminosidad es un claro ejemplo de los factores que afectan a la imagen; ésta generalmente afecta el contraste y la claridad de la imagen, ya que presentan acumulaciones de pixeles con niveles cromáticos altos o bajos.

Además de lo anterior, se tiene el problema de que cada vez la resolución de las imágenes es mayor (Fig. 1), por lo que el tiempo de procesamiento para mejorar este tipo de imágenes tiende a crecer, lo cual no es satisfactorio cuando hablamos de procesar cientos de miles de imágenes, como es el caso del área médica.

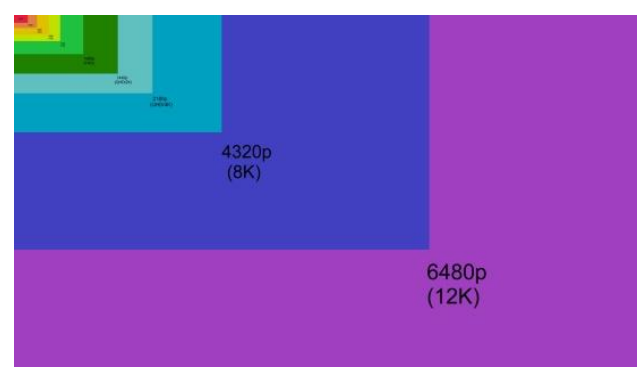

Fig. 1. Evolución de la resolución en imágenes digitales [2].

\subsection{Intentos anteriores - estado del arte}

Actualmente existe una gran variedad de técnicas que tratan este problema; uno de los más utilizados es la Ecualización del Histograma $(\mathrm{EH})$, por su fácil implementación y buenos resultados. El histograma de una imagen es una función discreta que representa el número de pixeles en la imagen en función al nivel de intensidad [3]. El objetivo de la Ecualización del Histograma es distribuir de forma uniforme el valor que toma cada uno de los pixeles a lo largo del rango dinámico, obteniendo como resultado una imagen con mejor contraste. Al ser secuencial, el tiempo de procesamiento puede ser alto o prohibitivo para algunas aplicaciones, como es el caso médico.

Otra técnica tradicionalmente utilizada es Power-LawTransform (PLT) [4], siendo una técnica rápida y sencilla, pero sigue siendo relativamente ineficiente; sin embargo, siguen surgiendo nuevas técnicas y variaciones de las mismas, tal es el caso de Contrast 
Limited Adaptive Histogram Equalization (CLAHE), siendo una variación de EH, usado en [5], para mejorar imágenes submarinas.

A pesarde la existencia de diferentes técnicas para abordar este problema, se siguen investigando y desarrollando nuevas, ya que existen diferentes problemáticas a resolver como lo es la definición de las imágenes, siendo éste un factor a considerar en la calidad de una imagen; se requiere mayor resolución para obtener la mejor calidad posible. Actualmente existen distintos formatos y resolución [6]. Estas nuevas resoluciones aumentan el tamaño de las imágenes y con ello su tiempo de procesamiento, por lo que existe la necesidad de procesar en un tiempo aceptable toda esta información en la imagen que tiende a ir en aumento. Esto exige nuevas técnicas capaces de procesar la mayor cantidad de información en el menor tiempo posible y obteniendo buenos resultados.

En este sentido, otras técnicas no tradicionales utilizadas en el procesamiento de imágenes son las Redes Neuronales Artificiales (RNAs), que han entregado buenos resultados[7]. Las Redes Neuronales Pulsantes o Pulso-Acopladas (Pulse-Coupled Neural Network PCNN) son una técnica que se ha utilizado en el área de Visión Artificial; este paradigma se basa en gran medida en el modelo de Eckhorn, que parte de la frecuencia de activación de las neuronas de la corteza visual [8]. Esto les permite cumplir de cierto modo algunas características visuales humanas [9], a diferencia de otras técnicas más matemáticas que pueden encontrarse en [10].

Del modelo PCNN surgen otras variantes como lo es el Modelo de Intersección Cortical (Intersection Cortical Model ICM) que es una versión más simple y está especialmente diseñado para el procesamiento de imágenes de manera eficiente. Fue presentado como una herramienta para el procesamiento de imágenes que es computacionalmente más rápido que el modelo PCNN completo. La característica de la auto-onda le permite procesar una vecindad de pixeles de manera más cercana a como lo hace la visión biológica. Algunas de sus aplicaciones han sido en la detección de objetos [11], la detección de ruido aditivo y gaussiano [12] y la detección de bordes [13]. Este modelo ICM, al igual que el modelo PCNN, entrega imágenes binarias que poseen una especie de meta-información o meta-conocimiento de la imagen y que puede ser utilizada para mejorarla. La introducción de la Matriz de Tiempo en un modelo de Red Neuronal Pulsante se ha utilizado para mejorar distintas características de la imagen como se muestra en [12,13], ya que ésta da información sobre el tiempo de disparo de las neuronas, obteniendo así información adicional de la imagen.

\subsection{Propuesta de solución - aportación}

En este trabajo se emplea una ICM pero con una Matriz de Tiempo (MT) para obtener meta-información o meta-conocimiento de la intensidad luminosa de los pixeles y ciertas zonas en la imagen, logrando así modificar y mejorar los valores cromáticos de la imagen. Con esta meta-información se alcanza una mejor distribución a lo largo del rango dinámico, sin perder zonas de interés y evitando introducir ruido a la imagen, logrando de esta forma una imagen con un mejor contraste y luminosidad.

Por otra parte, se presenta una configuración en los parámetros del modelo ICM para obtener un mejor desempeño con la Matriz de Tiempo, así mismo se presenta una modificación en la Matriz de Pesos para reducir la propagación de la auto-onda. Finalmente se presentan los diferentes resultados obtenidos con dos modelos 
propuestos basados en ICM, que incluyen algunas variaciones en la normalización y pesos.

Este artículo está organizado de la siguiente manera: en la Sección dos se presenta el modelo utilizado y la Matriz de Tiempo; en la Sección tres se describen los algoritmos propuestos; en la Sección cuatro se presenta la experimentación y resultados obtenidos, en particular se describen las métricas de evaluación utilizadas y se muestran los resultados de la evaluación de los experimentos, que arrojan que lo propuesto es altamente competitivo al ser comparado contra otras técnicas actuales presentadas en la literatura especializada; en la quinta Sección se presenta una discusión y finalmente las conclusiones y trabajo futuro es mencionado en la Sección seis.

\section{Descripción del Modelo de Intersección Cortical (ICM)}

\subsection{Modelo básico ICM}

El modelo neuronal ICM minimiza el costo del cálculo, manteniendo a la vez la efectividad del modelo cortical cuando se aplica a imágenes. Se basa en los elementos comunes de varios modelos biológicos [14]. Es un modelo simplificado del modelo PCNN, siendo la Red Neuronal ICM un caso especial de la Red PCNN en la cual no hay neuronas de enlace (linking). La ICM sólo utiliza entradas de alimentación (feeding). Es decir, si la fuerza de enlace de la PCNN se establece en ceros, se convertirá en la ICM. En la Fig. 2, se muestra el diagrama de la ICM.

La señal $F_{i j}$ se obtiene mediante el producto del parámetro $f$ y la señal $F_{i j}$ dada en un estado anterior; más la ponderación de la última salida del vecindario de neuronas $Y_{k l}$ por una matriz $w_{i j k l}$ (en este caso $i, j$ se refiere a las coordenadas del pixel que se está analizando y que corresponde a una neurona de ICM, mientras que $k, l$ se refieren a los pixeles vecinos de $i, j$, por lo que la matriz $W$ propiamente es de tamaño $k, l$ ) y por el estímulo externo de entrada $S_{i j}$. El umbral dinámico $T_{i j}$ crece obedeciendo a $h$ cuando su salida se activa y mantiene su estado previo con una atenuación dada por $g$, lo cual da origen a la formación de pulsos. El modelo ICM se describe con las ecuaciones (1), (2) y (3).

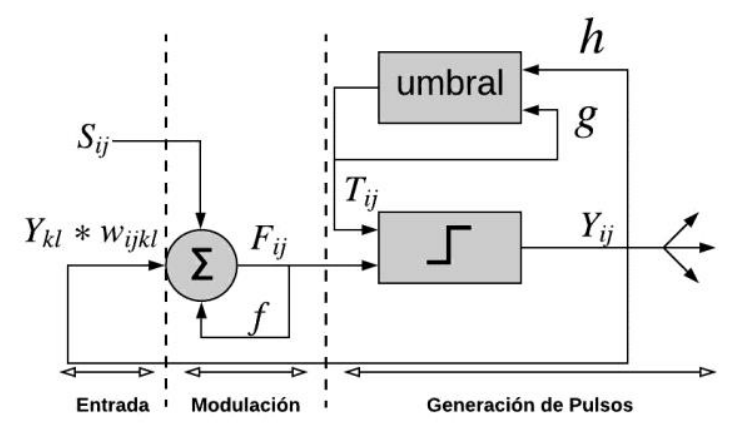

Fig. 2. Diagrama ICM [12]. 


$$
\begin{gathered}
F_{i j}[n]=f F_{i j}[n-1]+\sum w_{i j k l} Y_{k l}[n-1]+S_{i j}, \\
T_{i j}[n]=g T_{i j}[n-1]+h Y_{i j}[n-1], \\
Y_{i j}[n]=\left\{\begin{array}{cc}
1 & \text { si } F_{i j}[n]>T_{i j}[n] \\
0 & \text { en otro caso }
\end{array},\right.
\end{gathered}
$$

donde $n$ es la iteración actual, $w$ es la matriz de pesos sinápticos generalmente gaussiana de $3 \times 3$, como se muestra en la ecuación (4), que liga una neurona con sus vecinas y finalmente $f, g$ y $h$ son coeficientes de ajuste, donde $g<1.0, f<g$ y $h$ es un valor cercano a 20, según se recomienda en [14]:

$$
w_{k l}=\left[\begin{array}{ccc}
0.25 & 0.5 & 0.25 \\
0.5 & 0 & 0.5 \\
0.25 & 0.5 & 0.25
\end{array}\right]
$$

\subsection{ICM con Matriz de Tiempo o Meta-conocimiento}

En este artículo se emplea una ICM con una Matriz de Tiempo (MT) para obtener meta-información o meta-conocimiento de la intensidad luminosa de los pixeles además de algunas áreas específicas en la imagen. La MT se define para registrar la iteración o tiempo de activación para todas las neuronas $Y_{i j}$, como se muestra en la Fig. 3.

Si la neurona se dispara se registrará el tiempo en que se disparó [9]. Por tanto, un pequeño valor en la Matriz de Tiempo indica que el píxel correspondiente tiene una alta intensidad y pertenece a una región más brillante. De modo contrario, un gran valor indica que el píxel correspondiente tiene una intensidad baja y pertenece a una región más oscura. La Matriz de Tiempo se define con la ecuación (5).

$$
M_{i j}[n]=\left\{\begin{array}{cc}
n & \text { si } Y_{i j}=1, \\
M_{i j}[n-1] & \text { en otro caso. }
\end{array}\right.
$$

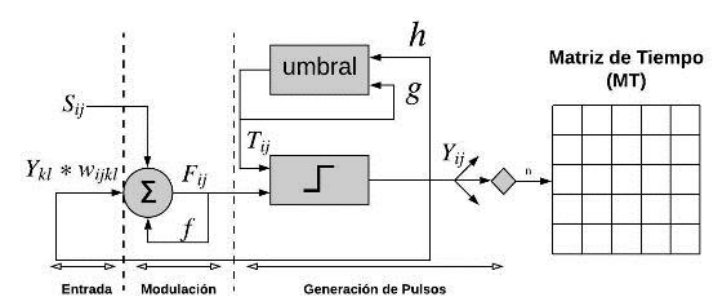

Fig. 3. Diagrama del modelo ICM-MT [12]. 


\section{Aportación: Modelos neuronales para mejorar imágenes médicas de gran resolución}

A continuación, se proponen y presentan dos modelos neuronales pulsantes diseñados para procesar eficientemente imágenes médicas de gran resolución que están, ó muy luminosas, o muy oscuras.

\subsection{Intersection Cortical Model - Luminance Time Matrix ICM-LTM}

El proceso del algoritmo propuesto denominado Modelo de Intersección Cortical con Matriz de Tiempo Luminosa (Intersection Cortical Model - Luminance Time Matrix ICM-LTM) se inspira en ICM, sólo que se anexa la Matriz de Tiempo donde se registrará el número de iteración $n$ de activación de cada una de las neuronas de salida $Y_{i j}$. El proceso del algoritmo se muestra en Fig. 4.

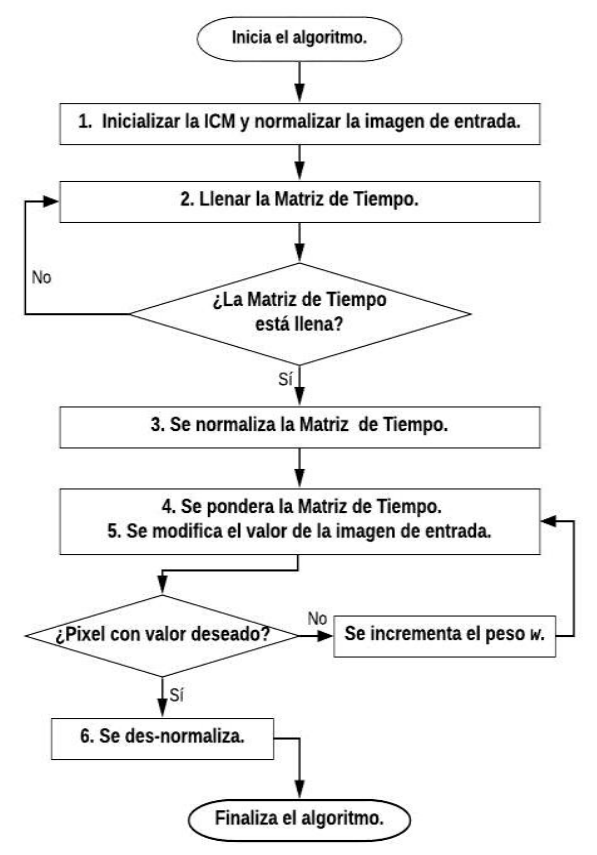

Fig. 4. Diagrama del modelo neuronal propuesto ICM-LTM.

Los seis pasos del algoritmo ICM-LTM se describen a continuación con un ejemplo de caso de imagen oscura.

Paso 1. El proceso del algoritmo inicia estableciendo los parámetros de la ICM, $f, g$ y $h$, del mismo modo se inicializan las matrices $F_{i j}, Y_{i j}, T_{i j}$ y $S_{i j}$, donde $S_{i j}$ se inicializa con los valores de la imagen de entrada normalizados, con respecto al valor máximo que puede tomar el pixel, en este caso al utilizar imágenes de 8 bits (255). Es decir, su 
valor original dividido entre 255 , obteniendo así una matriz con valores entre cero y uno como se muestra en Fig. 5.

Paso 2. El proceso para obtener la Matriz de Tiempo $M_{i j}$ es mostrado en la Fig. 6. Cuando la salida $Y_{i j}$ de la ICM es activada por primera vez se registra en la MT la iteración de activación. La ICM itera hasta conseguir que se disparen todas las neuronas. Esto da como resultado una MT con valores entre uno y el número de iteraciones requeridas para activar todas las neuronas (11 en este ejemplo), donde uno corresponde a las neuronas que se dispararon en la primera iteración y 11 corresponden a las últimas neuronas en activarse. Las primeras neuronas en activarse corresponden a los pixeles más luminosos y las últimas corresponden a los pixeles más oscuros.

Paso 3. La MT se normaliza dividiendo el valor en la MT y las iteraciones requeridas, restándole a ambas partes la primera época de activación; así la MT normalizada (MTN) queda con valores entre cero y uno (Fig. 7); con imágenes oscuras se modifica el valor restando uno al valor de la MT normalizada (Fig. 8).

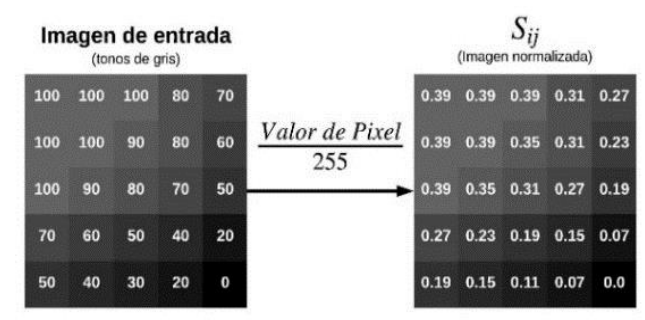

Fig. 5. Normalización de la imagen de entrada.

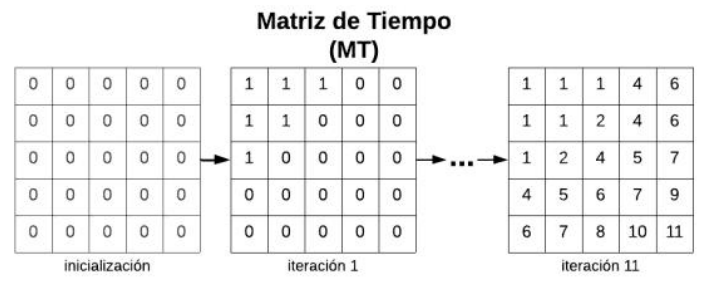

Fig. 6. Llenado de la Matriz de Tiempo.

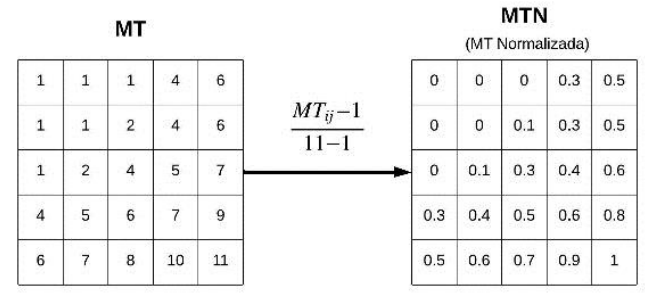

Fig. 7. Normalización de MT. 


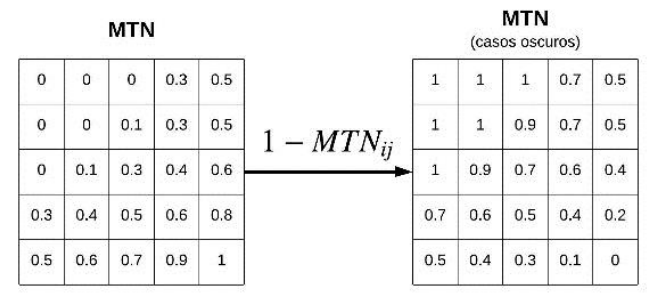

Fig. 8. MT normalizada (MTN) para imágenes oscuras.

Paso 4. Posteriormente, los valores de la Matriz de Tiempo normalizados se multiplican por un peso $w$ inicialmente de 0.1 para todos los pixeles con la finalidad de no modificar demasiado los valores de la imagen de entrada, obteniendo una Matriz de Tiempo ponderada (MTP) como se muestra en la Fig. 9.

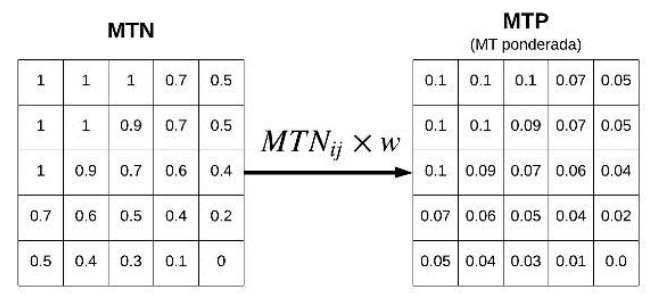

Fig. 9. Obtención de la MT ponderada (MTP).

Paso 5. El valor obtenido de esta multiplicación se utiliza para disminuir los valores de los pixeles de la imagen de entrada normalizada, en el caso de las imágenes luminosas, o incrementar en caso de las imágenes oscuras, es decir, a la matriz $S_{i j}$ se le resta o suma la MT ponderada, obteniendo como resultado el nuevo valor normalizado del pixel, la Fig. 10 muestra el resultado de una imagen oscura.

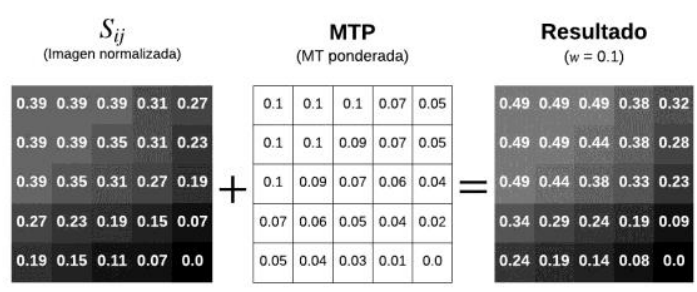

Fig. 10. Resultado $w=0.1$.

El proceso de obtención de la Matriz Ponderada se repite modificando el valor del peso $w$ hasta conseguir que por lo menos el valor de un pixel llegue al valor deseado, en este caso al ser una imagen oscura que cuenta con valores en cero se busca el uno (Fig. 11). Esto es con la finalidad de abarcar todo el rango dinámico de la imagen, siendo uno equivalente a 255 al des-normalizar. 


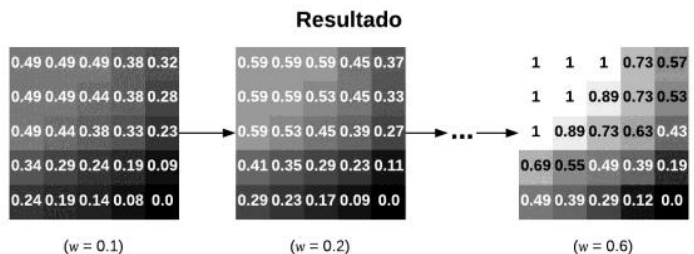

Fig. 11. Mejoramiento gradual del resultado.

Paso 6. Finalmente, el valor obtenido como resultado se des -normaliza multiplicándolo por 255, dando como resultado la imagen mejorada final (Fig. 12). La comparación de la imagen mejorada y la resultante de este ejemplo es mostrada en la Fig. 13.

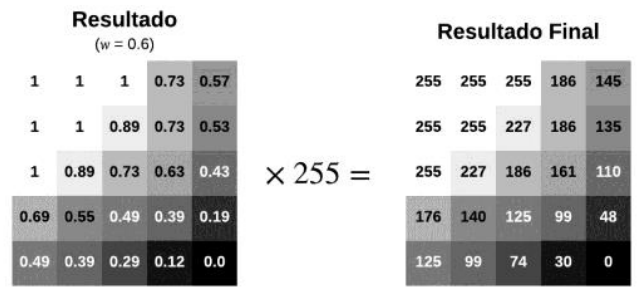

Fig. 12. Des-normalización de la imagen.

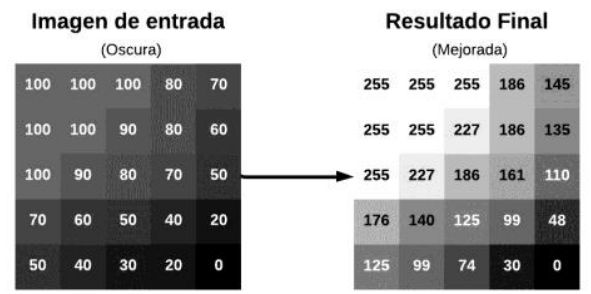

Fig. 13. Comparación de la entrada oscura y la salida mejorada.

\subsection{Intersection Cortical Model - Luminance Time Matrix2 ICM-LTM2}

El modelo que se denominó ICM-LTM2 es una modificación al algoritmo ICMLTM, con la finalidad de mejorar en tiempo y resultados. Se realizaron tres modificaciones: dos en el Paso uno, específicamente en la matriz de pesos de ICM y la normalización de la imagen de entrada y una modificación más en el Paso 4, donde se eliminaron las iteraciones para encontrar el peso adecuado. La primera modificación se realizó en la matriz de pesos, con el fin de reducir la onda expansiva de la ICM, ya que esto provocaba un destello en los bordes lo que afecta su calidad.

La segunda modificación se emplea en la normalización de la imagen de entrada normalizándola de acuerdo al rango dinámico de la imagen, es decir de acuerdo con el valor máximo que aparece en la imagen y el valor mínimo en la escala de 0 a 255 , 
logrando así reducir el tiempo requerido para obtener la MT. Finalmente se calculó directamente el peso $w$ requerido, estableciéndolo igual al valor mínimo en la imagen de entrada para imágenes luminosas y como la diferencia entre uno y el valor máximo de la imagen de entrada para imágenes oscuras; esto se realizó ya que el valor máximo que toma la MT ponderada es igual a uno y esto afectará más a los valores máximos para obtener un 255 a la salida en imágenes oscuras, o a los mínimos para obtener un cero en la salida en imágenes luminosas según sea el caso. Logrando de esta manera eliminar las iteraciones realizadas para encontrarlo y con ello reducir el tiempo de procesamiento.

\section{Experimentación y resultados}

Para la experimentación se tomaron tres estudios de resonancia magnética con 150 imágenes cada uno en tres modalidades: PD, T1 y T2, simuladas en BrainWeb [15-17]. Estas imágenes fueron desplazadas utilizando la técnica de Desplazamiento del Histograma (DH) [3], en: 20, 40, 60, 80, 100, 120 y 140 del valor cromático del pixel, tanto positivo como negativo, obteniendo así un total de 6,300 imágenes para la experimentación. Se establecieron los valores de $M_{i j}, Y_{i j}$ y $F_{i j}$, en cero, del tamaño de las imágenes de experimentación. La matriz $T_{i j}$ se inicializó en 1, del mismo tamaño que las imágenes de entrada. El parámetro $f$ se estableció en $0.1, g$ en 0.9 y $h$ en 20 tanto para ICM-LTM como para ICM-LTM2. Los parámetros fueron seleccionados de experimentaciones previas considerando la información que entregaba cada iteración de la ICM con respecto a la intensidad de los pixeles, el tiempo y distribución de los valores de la Matriz de Tiempo. La experimentación y los resultados se dividieron en tres etapas: la etapa 1), donde se realizaron experimentos con imágenes en modalidad PD; la etapa 2), donde se realizaron experimentos con la modalidad T1; y finalmente la etapa 3), donde se experimentó con imágenes en modalidad T2.

Los métodos utilizados para el mejoramiento de la imagen dependen generalmente de las necesidades especiales para algunas aplicaciones. En la mayoría de los casos, los efectos de mejora son evaluados por la percepción visual, difícilmente se llegan a evaluar de forma objetiva por medio de una métrica, a pesar de ello, existen diferentes métricas para evaluar la calidad de una imagen tomando en cuenta nitidez, contraste, color, claridad y artefactos. Se han usado diferentes modelos multidimensionales para evaluar la calidad a partir de las medidas obtenidas sobre los atributos; la calidad en este tipo de modelos se puede aproximar como una función que calcula la distancia euclídea de las imágenes degradadas con respecto a una imagen ideal en un espacio $n$ dimensional, como se muestra en la ecuación (6) [18]:

$$
C(x, y)=\sqrt{\sum_{i=1}^{n}\left(\left(x_{i}-y_{i}\right) * p_{i}\right)^{2}},
$$

donde la calidad $C$ es entendida como la distancia euclidiana entre una imagen digital $x$ y su imagen referente ideal $y$, a partir de sus atributos $i$, ponderados mediante sus coeficientes de ponderación $p$.

Otro método utilizado es la Métrica de Similitud Estructural (SSIM) que se usa para medir la similitud entre dos imágenes considerando la luminancia, contraste y 
estructura, combinando las tres y simplificando se obtiene la ecuación (7)[19]. Es una métrica de referencia completa por lo que toma una imagen de referencia siendo ésta la imagen sin distorsionar:

$$
\operatorname{SSIM}(x, y)=\frac{\left(2 \mu_{x} \mu_{y}+C_{1}\right)\left(2 \sigma_{x y}+C_{2}\right)}{\left(\mu_{x}^{2}+\mu_{y}^{2}+C_{1}\right)\left(\sigma_{x}^{2}+\sigma_{y}^{2}+C_{2}\right)}
$$

donde $\mu_{x}$ y $\mu_{y}$ son el promedio de cada una de las imágenes, $\sigma_{x}^{2}$ y $\sigma_{y}^{2}$ es la varianza de las imágenes, $\sigma_{x y}$ es la covarianza, $C_{1}=\left(k_{1} L\right)^{2}$ y $C_{2}=\left(k_{2} L\right)^{2}$, donde $L=255, k_{1}=$ 0.01 y $k_{2}=0.03$ por defecto.

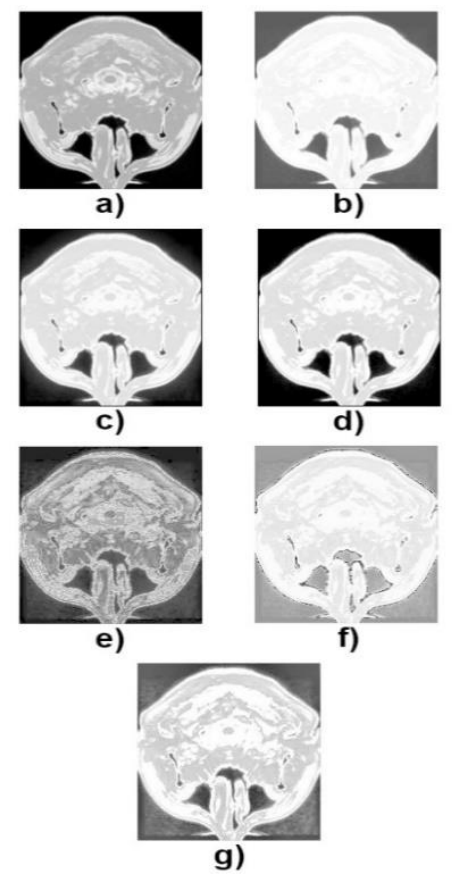

Fig. 14. Resultados PD luminosa, a) imagen original, b) imagen desplazada en +60 , resultados con los algoritmos, c) ICM-LTM, d) ICM-LTM2, e) EH, f) PLT, g) CLAHE.

Se iniciaron los experimentos con imágenes médicas en la modalidad PD, comenzando con las imágenes luminosas; se aplicaron los 7 desplazamientos antes mencionados de forma positiva en las 150 imágenes del estudio. Las imágenes fueron procesadas por los algoritmos: ICM-LTM, ICM-LTM2, EH [3], PLT[4], CLAHE[5]. Uno de los resultados obtenidos en esta experimentación se muestra en la Fig. 14, donde se observa que las imágenes obtenidas por los modelos propuestos visualmente se asemejan más a la imagen original, al igual que CLAHE, sin embargo, a pesar de entregar un buen contraste, éste presenta más ruido en la imagen resultante. Algo similar se hizo para las modalidades T1 y T2.

Los resultados de la evaluación de las tres etapas de experimentación antes mencionadas se muestran a continuación, considerando el promedio de las 150 imágenes de cada estudio en cada uno de los siete diferentes desplazamientos del 
histograma (DH), con las cinco técnicas empleadas, ICM-LTM, ICM-LTM2, EH, PLT y CLAHE evaluadas con la métrica $C$, que presenta un mejor resultado mientras más cercano a cero (Tabla 1) y SSIM, que se entiende como mejor resultado el más cercano a uno (Tabla 2); la evaluación general considera el promedio de los tres estudios en cada uno de los desplazamientos con las dos métricas utilizadas. Los mejores resultados evaluados según las métricas son marcados en negritas subrayadas, y los peores en cursivas subrayadas. Las Figs. 15 y 16 muestran esto de manera gráfica.

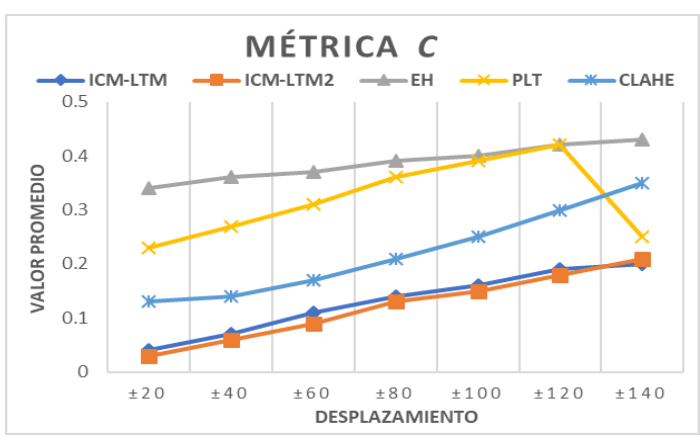

Fig. 15. Gráfica evaluación $C$.

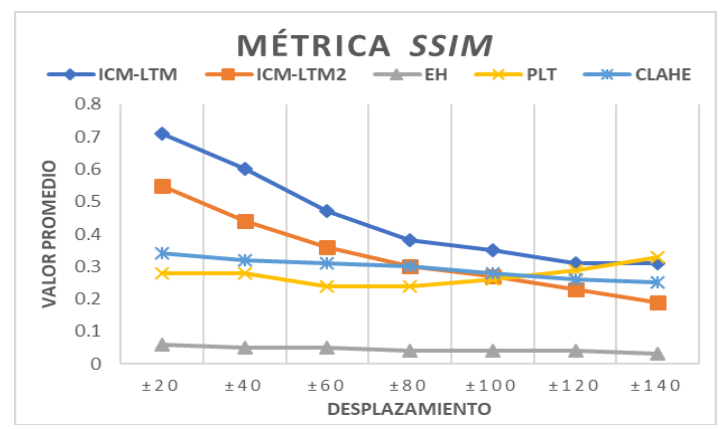

Fig. 16. Gráfica evaluación SSIM .

Tabla 1. Evaluación general con la métrica $C$.

\begin{tabular}{c|ccccc}
\hline DH & ICM-LTM & ICM-LTM2 & EH & PLT & CLAHE \\
\hline 20 & 0.04 & $\underline{\mathbf{0 . 0 3}}$ & $\underline{0.34}$ & 0.23 & 0.13 \\
40 & 0.07 & $\underline{\mathbf{0 . 0 6}}$ & $\underline{0.36}$ & 0.27 & 0.14 \\
60 & 0.11 & $\underline{\mathbf{0 . 0 9}}$ & $\underline{0.37}$ & 0.31 & 0.17 \\
80 & 0.14 & $\underline{\mathbf{0 . 1 3}}$ & $\underline{0.39}$ & 0.36 & 0.21 \\
100 & 0.16 & $\underline{\mathbf{0 . 1 5}}$ & $\underline{0.40}$ & 0.39 & 0.25 \\
120 & 0.19 & $\underline{\mathbf{0 . 1 8}}$ & $\underline{0.42}$ & $\underline{0.42}$ & 0.30 \\
140 & $\underline{\mathbf{0 . 2 0}}$ & 0.21 & $\underline{0.43}$ & 0.25 & 0.35 \\
\hline Prom. & 0.13 & $\underline{\mathbf{0 . 1 2}}$ & $\underline{0.39}$ & 0.32 & 0.22 \\
\hline
\end{tabular}


Tabla 2. Evaluación general con la métrica SSIM.

\begin{tabular}{c|ccccc}
\hline DH & ICM-LTM & ICM-LTM2 & EH & PLT & CLAHE \\
\hline 20 & $\underline{\mathbf{0 . 7 1}}$ & 0.55 & $\underline{0.06}$ & 0.28 & 0.34 \\
40 & $\underline{\mathbf{0 . 6 0}}$ & 0.44 & $\underline{0.05}$ & 0.28 & 0.32 \\
60 & $\underline{\mathbf{0 . 4 7}}$ & 0.36 & $\underline{0.05}$ & 0.24 & 0.31 \\
80 & $\underline{\mathbf{0 . 3 8}}$ & 0.30 & $\underline{0.04}$ & 0.24 & 0.30 \\
100 & $\underline{\mathbf{0 . 3 5}}$ & 0.27 & $\underline{0.04}$ & 0.26 & 0.28 \\
120 & $\underline{\mathbf{0 . 3 1}}$ & 0.23 & $\underline{0.04}$ & 0.29 & 0.26 \\
140 & 0.31 & 0.19 & $\underline{0.03}$ & $\underline{\mathbf{0 . 3 3}}$ & 0.25 \\
\hline Prom. & $\underline{\mathbf{0 . 4 5}}$ & 0.33 & $\underline{0.04}$ & 0.27 & 0.29 \\
\hline
\end{tabular}

\section{Conclusiones y trabajo futuro}

Se obtuvo en promedio un $10 \%$ de mejora con la métrica $C$ y un $4 \%$ de mejora con SSIM en comparación con CLAHE, siendo esta la técnica que entregó mejores resultados en promedio. Se observó que las técnicas entregan un mejor desempeño en imágenes luminosas que en imágenes oscuras lo que podría deberse a las modificaciones realizadas para tratar con ellas.

La modificación realizada en ICM-LTM2 ayudó a obtener los resultados en menor tiempo hasta en un $25 \%$ más rápido y entregar mejores resultados con respecto a la métrica $C$.

Como trabajo futuro podrían optimizarse los parámetros de la ICM al igual que la Matriz de Pesos $w$, ya que han sido establecidas de acuerdo a las propuestas en la literatura y experimentos realizados a prueba y error. Así mismo, habrá que realizar experimentos estableciendo el valor deseado mínimo y máximo del pixel de acuerdo al tipo de estudio, ya que el exceso de brillo o falta de él, podría deberse a que la imagen naturalmente no toma valores en 0 ó 255; del mismo modo, trabajar la implementación del algoritmo en paralelo para aprovechar las ventajas que presenta su naturaleza paralela, estimándose ahorros en tiempo en aproximadamente $n$ unidades siendo éste igual al número de núcleos utilizados y menor o igual al número de pixeles en la imagen a procesar. En todo caso, lo presentado y propuesto resulta una alternativa muy competitiva para procesar imágenes de gran resolución.

\section{Referencias}

1. Pedersen, M. Bonnier, N. Hardeberg, J.Y. Albregtsen, F.: Attributes of image quality for color prints. J. Electron. Imaging 19(1), 011016.doi:10.1117/1.3277145 (2010)

2. Wikimedia Commons (s.f.): https://commons.wikimedia.org/wiki/File:12 K_ Screen_Resolution_Column.jpg Último acceso: 2019/04/01

3. Pajares, G., Cruz, J.M. de la.: Ejercicios resueltos de visión por computador. Madrid: Alfaomega (2008) 
4. Janani, P., Premaladha, J., Ravichandran, K.S.: Image Enhancement Techniques: A Study. $\begin{array}{lllll}\text { Indian Journal of Science and Technology } 8(22), & 12 .\end{array}$ doi:10.17485/ijst/2015/v8i22/79318 (2015)

5. Ma, J., Fan, X., Yang, S.X., Zhang, X., Zhu, X.: Contrast Limited Adaptive Histogram Equalization-Based Fusion in YIQ and HSI Color Spaces for Underwater Image Enhancement. International Journal of Pattern Recognition and Artificial Intelligence 32(07), 1854018. doi:10.1142/S0218001418540186 (2018)

6. Castillo, J.M.: El futuro de la televisión. Comunicación y hombre: revista interdisciplinar de ciencias de la comunicación y humanidades (11), 83-100 (2015)

7. Jena, M., Mishra, S.: Review of Neural Network Techniques in the Verge of Image Processing. In: International Proceedings on Advances in soft computing, Intelligent Systems and Applications, pp. 345-361. Singapore: Springer, Singapore. doi:10.1007/978-981-105272-9_33 (2018)

8. Eckhorn, R., Reitboeck, H.J. Arndt, M., Dicke, P.: Feature Linking via Synchronization among Distributed Assemblies: Simulations of Results from Cat Visual Cortex. Neural Computation 2(3), 293-307. doi:10.1162/neco.1990.2.3.293 (1990)

9. Ma, Y., Zhan, K., Wang, Z.: Applications of pulse-coupled neural networks. Berlin: Springer (2010)

10. Rodríguez Morales, R., Sossa Azuela, J.H.: Procesamiento y análisis digital de imágenes. México: Alfaomega (2011)

11. Ekblad, U., Kinser, J.M., Atmer, J., Zetterlund, N.: The intersecting cortical model in image processing. Nuclear Instruments and Methods in Phy sics Research Section A: Accelerators, Spectrometers, Detectors and Associated Equipment, 525(1-2), 392-396. doi:10.1016/J.NIM A.2004.03.102 (2004)

12. Ortiz, E., Mejía-Lavalle, M., Sossa, H.: Using Pulse Coupled Neural Networks to Improve Image Filtering Contaminated with Gaussian Noise. Computación y Sistemas 21(2), pp. 381395. doi: 10.13053/Cy S-21-2-2742 (2017)

13. Cardenas, S.Y., Mejia-Lavalle, M., Sossa, H., Pardo, E C.: Pulse-Coupled Neural Networks applied to Human Brain Image Processing. In: 2014 International Conference on mechatronics, electronics and automotive engineering, pp. 60-65. IEEE. doi:10.1109/ICMEAE.2014.46 (2014)

14. Lindblad, T., Kinser, J.M.: Image processing using pulse-coupled neural networks. Heidelberg New York Dordrecht London: Springer (2005)

15. Kwan, R.K.-S., Evans, A.C., Pike, G.B.: An Extensible MRI Simulator for Post-Processing Evaluation. Visualization in Biomedical Computing 1131, 135-140 (1996)

16. Kwan, R.K.-S., Evans, A.C., Pike, G.B.: MRI simulation-based evaluation of imageprocessing and classification methods. IEEE Transactions on medical imaging 18(11), 108597 (1999)

17. Collins, D.L., Zijdenbos, A.P., Kollokian, V., Sled, J.G., Kabani, N.J., Holmes, C.J., Evans, A.C.: Design and Construction of a Realistic Digital Brain Phantom. IEEE Transactions on Medical Imaging 17(3), 463-468 (1998)

18. Robledano-Arillo, J., Moreno-Pelay o, V., Pereira-Uzal, J.M .: Aproximación experimental al uso de métricas objetivas para la estimación de calidad cromática en la digitalización de patrimonio documental gráfico. Revista Española de Documentación Científica 39(2), e128. doi:10.3989/redc.2016.2.1249 (2016)

19. Wang, Z., Bovik, A.C., Sheikh, H.R., Simoncelli, E.P.: Image Quality Assessment: From Error Visibility to Structural Similarity. IEEE Transactions on image processing 13(4), 114 (2004) 